\title{
Neural correlates of own- and other-race face recognition in children: A functional near-infrared spectroscopy study
}

\author{
Xiao Pan Ding ${ }^{1}$, Genyue Fu ${ }^{1}$, and Kang Lee ${ }^{1,2}$ \\ ${ }^{1}$ Zhejiang Normal University, China \\ ${ }^{2}$ University of Toronto, Canada
}

\section{Abstract}

The present study used the functional Near-infrared Spectroscopy (fNIRS) methodology to investigate the neural correlates of elementary school children's own- and other-race face processing. An old-new paradigm was used to assess children's recognition ability of own- and other-race faces. FNIRS data revealed that other-race faces elicited significantly greater [oxy-Hb] changes than own-race faces in the right middle frontal gyrus and inferior frontal gyrus regions (BA9) and the left cuneus (BA18). With increased age, the [oxy-Hb] activity differences between own- and other-race faces, or the neural other-race effect (NORE), underwent significant changes in these two cortical areas: at younger ages, the neural response to the other-race faces was modestly greater than that to the own-race faces, but with increased age, the neural response to the own-race faces became increasingly greater than that to the other-race faces. Moreover, these areas had strong regional functional connectivity with a swath of the cortical regions in terms of the neural other-race effect that also changed with increased age. We also found significant and positive correlations between the behavioral other-race effect (reaction time) and the neural otherrace effect in the right middle frontal gyrus and inferior frontal gyrus regions (BA9). These results taken together suggest that children, like adults, devote different amounts of neural resources to processing own- and other-race faces, but the size and direction of the neural other-race effect and associated functional regional connectivity change with increased age.

\section{Keywords}

fNIRS; face processing; face recognition; the other-race effect; own-race or other-race face recognition; functional connectivity; children; development

Human adults are experts at processing faces. We are able to discriminate very subtle differences between two faces (e.g., Ge et al., 2009), and readily recognize thousands of faces, many of which have not even been seen for decades (e.g., Bahrick, Bahrick, \& Wittlinger, 1975). Nevertheless, our face expertise is only limited to the category of faces with which we have extensive experience. One prime example of such limitation is the socalled other-race effect (ORE), whereby we have greater recognition memory for own-race faces than that for other-race faces due to our extensive experience with individuals from our own race, and limited or no experience with individuals from other races. This effect is robust in adults and has been found with the use of various methodologies and with adults

Crown Copyright $\odot 2013$ Published by Elsevier Inc. All rights reserved.

Publisher's Disclaimer: This is a PDF file of an unedited manuscript that has been accepted for publication. As a service to our customers we are providing this early version of the manuscript. The manuscript will undergo copyediting, typesetting, and review of the resulting proof before it is published in its final citable form. Please note that during the production process errors may be discovered which could affect the content, and all legal disclaimers that apply to the journal pertain. 
from different racial backgrounds (Hancock \& Rhodes, 2008; Hayward, Rhodes, \& Schwaninger, 2008; Mondloch et al., 2010; Pezdek, Blandon-Gitlin, \& Moore, 2003; Rhodes, Hayward, \& Winkler, 2006; Sangrigoli, Pallier, Argenti, Ventureyra, \& de Schonen, 2005; Walker \& Tanaka, 2003; for reviews, see Hugenberg, Young, Bernstein, \& Sacco, 2010; Meissner \& Brigham, 2001).

Developmental research has revealed that the other-race effect emerges in infancy (for a review, see Lee, Anzures, Quinn, Pascalis, \& Slater, 2011, and Anzures, Quinn, et al., in press). By three months of age, infants who only have experience with own-race individuals prefer to look at own-race faces over other-race faces (Bar-Haim, Ziv, Lamy, \& Hodes, 2006; Kelly et al., 2005). At 6 months, infants begin to show a recognition advantage for own-race faces, and by 8-9 months of age this other-race effect becomes more pronounced (Anzures, Quinn, Pascalis, Slater, \& Lee, 2010; Ferguson, Kulkofsky, Cashon, \& Casasola, 2009; Hayden, Bhatt, Joseph, \& Tanaka, 2007; Hayden, Bhatt, Zieber, \& Kangas, 2009; Kelly et al., 2007, 2009; Sangrigoli \& de Schonen, 2004a ). With further experience with own-race faces and continued lack of experience with other-race faces, children have also been found to have an own-race face recognition advantage with increased age, with the other-race effect less reliable at younger ages and becoming more robust with increased age (Chance, Turner, \& Goldstein, 1982; Feinman \& Entwisle, 1976; Goodman et al., 2007; Pezdek et al., 2003; Sangrigoli \& de Schonen, 2004b; Walker \& Hewstone, 2006; Anzures, Kelly et al., in press). The existing behavioral findings from infants, children, and adults taken together strongly support the proposal (Scott, Pascalis, \& Nelson, 2007) that our asymmetrical experience with different types of faces (e.g., own- versus other-race faces or own- versus other- species faces) affects the development of face processing expertise in a profound manner.

In contrast to the large number of behavioral studies, limited event-related potential (ERP) and functional magnetic resonance (fMRI) studies have examined the neural mechanisms underlying the other-race effect of face processing in adults (for a review, see Ito \& Bartholow, 2009). Using the ERP technique, which has exquisite temporal resolution but poor spatial resolution, recent studies (Caharel et al., 2011; Caldara, Rossion, Bovet, \& Hauert, 2004; Ito \& Urland, 2005) have revealed a number of marked electrophysiological response differences as early as $170 \mathrm{~ms}$ post stimulus onset when adults view own- and other-race faces. Using the fMRI technique, which has high spatial resolution but poor temporal resolution, researchers have also found that adults show blood hemoglobin response differences between own- and other-race faces in various cortical and subcortical regions such as the amygdala, bilateral fusiform gyrus (e.g., the Fusiform Face Area or FFA: Golby, Gabrieli, Chiao, \& Eberhardt, 2001; Kanwisher \&Yovel, 2006; Natu, Raboy, \& O'Toole, 2010), middle occipital gyrus (e.g., the occipital face area or OFA: Kanwisher \& Yovel, 2006; Natu et al., 2010), and bilateral prefrontal cortical regions such as the inferior frontal gyrus, and middle frontal gyrus (Cunningham et al., 2004; Feng et al., 2011; Kim et al., 2006; Lieberman, Hariri, Jarcho, Eisenberger, \& Bookheimer, 2005; Van Bavel, Packer, $\&$ Cunningham, 2008). These adult findings thus reveal a neural other-race effect (NORE) paralleling the behavioral other-race effect, suggesting that adults' asymmetrical experience with own- and other-race faces have a direct impact not only on their behavior but also on neural responses.

However, the developmental origin of the neural other-race effect is entirely unknown. Except for one ERP study demonstrating infants to respond differentially to own- and otherrace faces (Balas, Westerlund, Hung, \& Nelson, 2011), to the best of our knowledge, no neuroimaging study to date has investigated the neural correlates of own- and other-race face processing in children. Neuroimaging research on the development of the other-race effect will bridge the significant gap in our knowledge about how the neural other-race 
effect emerges and develops in childhood. This knowledge will thus bring us closer to a comprehensive understanding of the development of own- and other-race face processing from infancy to adulthood at both behavioral and neural levels. Further and more broadly, such research will elucidate how experience influences the developing brain systems to acquire such important visual expertise as face processing abilities.

To bridge this significant gap in the literature, we conducted the present study with Chinese children between the ages of 7 and 13 years who had been exposed nearly exclusively to own-race faces and had no direct contact with other-race individuals. We used an old-new paradigm to assess children's recognition ability of own- and other-race faces. Children were first asked to remember a set of Chinese faces in the Chinese Face Recognition task or Caucasian faces in the Caucasian Face Recognition task. After familiarization, they were shown the studied "old" (target) faces mixed with an equal number of new, unstudied (foil) Chinese or Caucasian faces. They judged which face was old and which was new.

We used the functional Near-infrared Spectroscopy (fNIRS) methodology to investigate the neural correlates of children's own- and other-race processing. Although NIRS does not have as high of a spatial resolution as the MRI methodology and only measures cortical hemodynamic activities a few centimeters deep in the cortex, it has several advantages, especially for the purpose of the present study. First, a NIRS machine is quiet and highly mobile and does not require a specially controlled laboratory environment. Thus, experiments can take place in an environment that is not as foreign, noisy, dark, or claustrophobic as that of an MRI machine (Suda et al., 2010). Second, NIRS has much higher temporal resolution than MRI in a typical fMRI study, at $10 \mathrm{~Hz}$ (although recent advances in fMRI protocols may soon mitigate this advantage). This temporal resolution makes it possible to obtain a recording of the actual time course of one hemodynamic response epoch in response to a specific cognitive task in a specific trial when a slow eventrelated design is used. Third, unlike an fMRI experiment where participants must lie down on a gantry with their head firmly restrained in a head coil, participants in an fNIRS experiment can sit up in a normal posture with less head restraining due to NIRS's relative higher tolerance for motion. Forth, due to the small operating costs associated with NIRS, it is possible to run fNIRS study with a large sample of participants of a wide age span. Thus, fNIRS is particularly well suited for developmental studies involving children.

Based on the existing adult findings (e.g., Feng et al., 2011), we hypothesized that own- and other-race faces would engender different levels of neural responses. The activation differences should be observed in areas of the posterior occipital cortex such as the middle occipital gyrus (MOG) and areas of the prefrontal cortex such as the inferior frontal gyrus (IFG) and the middle frontal gyrus (MFG). These areas have been implicated in existing adult fMRI studies of face processing in general (e.g., Esterman \& Yantis, 2010; Li et al., 2010; Maurer et al., 2007) and own- and other-race face processing in specific (e.g., Cunningham et al., 2004; Feng et al., 2011; Lieberman et al., 2005). The prefrontal areas are known to be part of the extended face processing network in adults (Haxby, Hoffman, \& Gobbini, 2000). We also hypothesized that with increased age, children's differential neural responses to own- and other-race faces would become increasingly greater in both occipital and prefrontal regions. Further, children's behavioral responses to own- and other-race faces would be significantly correlated with their differential neural responses to the two types of faces. 


\section{Methods}

\section{Participants}

Seventy-two Chinese children with no history of neurological or psychiatric disorders took part in this study (Mean age $=9.98, \mathrm{SD}=1.79$, range from 7.13 to 13.50 years; 40 boys, 32 girls). All children were Han Chinese living in a metropolitan city with $99.99 \%$ of the population being Han Chinese. They all had normal or corrected to normal vision. None of them reported having direct contact with any other-race individuals. The research was approved by the university ethics committee. Informed consent was obtained from all parents prior to beginning the study and oral assent was obtained from all child participants. After the experiment, children were debriefed and given a prize (e.g., toys).

\section{Stimuli}

Black-and-white photos of 20 Chinese and 20 Caucasian young female adults with a resolution of $450 \times 600$ pixels were used in the present study. All faces were upright, frontal with neutral emotional expressions.

\section{Procedure}

Children were seen individually. They were seated in front of a computer screen at a distance of $60 \mathrm{~cm}$. They were instructed to complete two within-subjects tasks: the Chinese face task and the Caucasian face task. Each task included two phases: the learning phase and the testing phase (Figure 1). During the learning phase, children were shown 10 target faces (Chinese or Caucasian) in a randomized order and asked to remember all of the faces. The faces were shown three times. During each trial of the learning phase, each face was presented for $3000 \mathrm{~ms}$ with intervals of a $2000 \mathrm{~ms}$ central fixation point between two adjacent face photos. During the testing phase, the 10 target face photos that were used in the learning phase were intermixed randomly with another 10 foil faces (Chinese or Caucasian). The testing target and foil faces were shown individually. Each face was displayed for $3000 \mathrm{~ms}$ after a $2000 \mathrm{~ms}$ central fixation point, followed by another fixation point lasting $8000 \mathrm{~ms}$ in order to make sure the signal activated by the last trial returned to baseline. Children were asked to press either ' 1 ' or '2' on the keyboard to indicate whether the face presently seen was an "old" target face (one learned during the learning phase) or a "new" foil face. The key assignment was counterbalanced between children. The order of Chinese task or Caucasian task was counterbalanced between children. During the testing phase, we used the NIRS imager to measure children's neural responses to own- and otherrace faces.

\section{fNIRS data acquisition}

A 48 channels continuous wave system (ETG-4000, Hitachi Medical Co., Japan) was used in the present study (although we only used 46 channels). The probes of the NIRS machine were fixed using two $6 \times 6 \mathrm{~cm}^{2}$ rubber shells over the frontal areas and one $6 \times 12 \mathrm{~cm}^{2}$ rubber shell over the occipital areas. The shells were covered with a nylon-net to keep them attached to the head. The two shells of 9 probes, consisting of a pair of $3 \times 3$ arrays with five light emitters and four detectors, and one shell of 15 probes, consisting of a $5 \times 3$ arrays with eight light emitters and seven detectors, were capable of measuring the relative concentrations of hemoglobin at 46 points (See Figure 2). The lowest probes of the frontal areas were positioned along the Fp1-Fp2 line while the lowest probes of the occipital areas were positioned along the $\mathrm{O} 1-\mathrm{O} 2$ line in accordance with the international 10-20 system for electroencephalography. The placements of the probes in the dorsal bilateral frontal areas and occipital areas were based on our hypotheses derived from the existing related fMRI studies. The inter-optode distance was $30 \mathrm{~mm}$, which allowed for measuring hemodynamic 
activities approximately $15-25 \mathrm{~mm}$ beneath the scalp. Optical data from individual channels were collected at 2 different wavelengths $(695$ and $830 \mathrm{~nm})$ and analyzed using the modified Beer-Lambert Law for a highly scattering medium (Cope \& Delpy, 1988). Changes in oxygenated $([\mathrm{oxy}-\mathrm{Hb}])$ and deoxygenated $([\mathrm{deoxy}-\mathrm{Hb}])$ signals were calculated in units of millimolar-millimeter (mM*mm) (Maki et al., 1995). The sampling rate was set to $10 \mathrm{~Hz}$.

We used a 3D-magnetic space digitizer (EZT-DM401, Hitachi Medical Corporation, Japan) to measure the exact spatial location of each optode in relation to the veridical landmarks of a participant's head (i.e., nasion, inion, $\mathrm{Cz}$, the pre auricular points anterior to the left and right ears). We used the probabilistic registration method (Singh et al., 2005) to obtain estimation of each channel's corresponding location in the Montreal Neurological Institute (MNI) space.

\section{fNIRS data analysis}

We analyzed the [oxy-Hb] and [deoxy-Hb] signals. For each participant, we segmented event epochs from the time course data. Each event epoch consisted of a $2 \mathrm{~s}$ period prior to stimulus onset, $3 \mathrm{~s}$ of the stimulus period, $5 \mathrm{~s}$ of the recovery period, and a $3 \mathrm{~s}$ post-stimulus period. However, we found the [deoxy-Hb] signals to be noisy and unreliable as reported by other researchers previously (Homae, Watanabe, Nakano, \& Taga, 2007). Thus, we focused our data analyses only on the [oxy-Hb] data here.

General linear model (GLM) analysis-To explore the spatial correlates underlying the different trial types, the [oxy-Hb] data were analyzed using NIRS-SPM (Jang et al., 2009; Tak et al., 2011; Ye et al., 2009). For each participant, data were preprocessed to remove sources of noise and artifact (such as movement and heart rate) using a hemodynamic response function (hrf) filter and a wavelet-MDL (minimum description length) detrending algorithm. Then, a general linear model incorporating task effects, a mean and a linear trend were used to compute parameter estimates and t-contrasts for the comparison (own-race faces recognition minus other-race faces recognition during the testing phase) at each channel. NIRS channel positions on an estimated MNI space were obtained from a 3D digitizer using algorithms by Singh et al. (2005).

\section{Regional functional connectivity analysis results based on the GLM data from} individual children-To further understand whether individual children's neural responsivity in one channel correlated with their responsivity in another, we first identified the channel that revealed significant differences between the own- and other-race face conditions based on the GLM analyses. Then, we use this channel as the seed channel to perform Pearson correlations between children's beta values derived from the GLM analyses in the seed channel with their beta values in all other channels.

Correlations between behavior and NIRS GLM data-To understand whether children's neural responsivity correlated with their behavioral responses, we calculated Pearson correlations between children's behavioral measures (the correct reaction time and accuracy in own-race and other-race faces conditions) and their beta values in all channels.

\section{Results}

\section{Behavior results}

Preliminary results revealed no significant effects for gender. Thus, the results for this factor were collapsed for all subsequent analyses. The descriptive results of face recognition performance are shown in Table 1. 
Three analyses of variance were first conducted to determine if there was an overall preference to one face type over the other. A repeated measures 2 (stimulus race: own vs. other race) $\times 1$ (age in years: continuous) ANOVA was performed with stimulus race (ownrace face or other-race face) as a within-subjects variable, participant age as a continuous variable, and accuracy and reaction time in milliseconds as the dependent variables. For the accuracy, only the main effect of age was significant, $F(1,70)=5.93, p<.05, \eta^{2}=.08$ : with increased age, children's accuracy improved significantly for both own- and other-race faces. For the correct reaction time, only the main effect of age was significant, $F(1,70)=$ $4.89, p<.05, \eta^{2}=.07$ : with increased age, children's reaction time decreased significantly. However, no significant differences were found between own- and other-race faces ( $p s>$. 05).

\section{fNIRS results}

GLM result-To investigate the spatial correlates underlying the ORE of faces, we compared the hemodynamic activities between the own- and other-race faces. Two repeated measures 2 (stimulus race: own-race vs. other-race face) $\times 1$ (age in years: continuous) ANOVAs were performed with stimulus race (own-race face or other-race face) as a withinsubjects variable, participant age as a continuous variable, and changes in changes in [oxy$\mathrm{Hb}$ ] signals as the dependent variables.

The results showed that the other-race faces elicited significantly larger changes in [oxy-Hb] signals than the own-race faces in Channel 19 (BA9, right MFG/IFG) and Channel 40 (BA18, left cuneus), indicating that recognizing other race faces engaged in significantly greater neural activities than recognizing own race faces in the frontal and occipital area (the results were corrected by FDR, $p<.05$ ) (See Table 2$)$.

Regarding the age effect, Pearson correlations were computed between age in years and the contrast beta values between own-race faces minus other-race faces in Ch19 and Ch40. Both correlations were significant $(r=.30, p<.05 ; r=.25, p<.05$; Figure 3a\&b). Thus, with increased age, the [oxy-Hb] activity differences between own- and other-race faces, or the neural other-race effect, increased significantly in Ch19 (right MFG/IFG) and Ch40 (left cuneus).

To further explore this age-related NORE obtained when we used the more sensitive age-inyears measure (continuous variable), we divided children into three age groups: 7.0 to 8.8 (Mean age=7.94, N=27), 8.8 to 11.7 (Mean age=10.63, $\mathrm{N}=29$ ), 11.7 to 13.5 (Mean age $=12.23, \mathrm{~N}=16$ ) years. They are henceforth referred to as Age Groups 1, 2, and 3, respectively. Three one-sample t-tests were conducted against the zero which reflects the lack of the neural other-race effect. We found that in Age Group 1, there were marginally negative NORE effects in Channel $19(t=-1.9, p=.058)$ and Channel $40(t=-2.1, p=.041)$. Thus, between 7.0 to 8.8 years, the neural response to the other-race faces was greater than that to the own-race faces (resulting in a negative difference score). In Age Group 2, the NORE was not significantly different from zero. In Age Group 3, there were marginally positive NORE in Channel $19(t=2.07, p=.056)$ but not Channel 40, indicating that between 11.7 and 13.5 years, the neural response to the own-race faces was greater than that to the other-race faces (resulting a positive difference score).

To determine whether the null NORE effect in Age Group 2 (from 8.8- to 11.7- year-old) was due to the fact that own-race face activity was rising or the other-race face activity was dropping relative to the younger children and the older children, one-way ANOVAs were conducted to compare the beta values of own-race faces or other-race faces in Ch19 and $\mathrm{Ch} 40$ in the three age groups, respectively. Results showed that there was a significant effect of age group on the beta values of own-race faces in $\operatorname{Ch} 19(F(2,69)=3.36, p<.05)$. However, 
there was no significant effect on that of other-race faces in either Ch19 or Ch40. Post-hoc tests showed that the mean score of neural activity of own-race faces in Ch19 for Age group $3(M=-.09, S D=.10)$ was significantly higher than Age group $1(M=.00, S D=.14)$ and Age group $2(M=.00, S D=.11)$, indicating that the own-race activity was rising but the otherrace face activity was not dropping with increased age.

Regional functional connectivity analysis results-To further examine whether individual children's neural responsivity in one channel correlated with their responsivity in another, we calculated the Pearson correlations between the contrast beta values of own-race faces minus that of other-race faces in Ch19 (i.e., the neural other-race effect in this channel) with the contrast beta values between own- and other-race faces in all other channels. We did the same for $\mathrm{Ch} 40$. The reason we chose these two channels as seed channels was that both showed a significant neural other-race effect (i.e., the [oxy-Hb] activity difference between own- and other-race faces) based on the GLM analyses.

We found that Ch19 was significantly and positively correlated with 11 cortical areas (Figure 4a). The bigger a child's neural other-race effect in Ch19, the greater the child's neural other-race effect in these 11 cortical areas. Most notably, the neural other-race effect in Ch19 was significantly and positively correlated with those in Ch40 and the other seed channel as well. To ascertain whether these significant correlations continued to exist after the age factor was controlled, we also computed partial correlations between Ch19 and all other channels after controlling for age. The same results were obtained except for the correlation between Ch19 and Ch40 (Figure 4b). Thus, after partialling out the effect of age, Ch19 appeared to have strong regional functional connectivity with a large swath of the cortical regions in terms of the neural other-race effect. For the correlation between Ch19 and $\mathrm{Ch} 40$, age appeared to be a mediating factor. Also, we found that $\mathrm{Ch} 40$ was significantly and positively correlated with 12 cortical areas (Figure 4c). After controlling for age, the same results were obtained, indicating the neural other-race effect to be functionally linked between $\mathrm{Ch} 40$ and these 12 cortical areas and to be robust after partialling out the effect of age (Figure 4d).

To further explore the age-related regional functional connectivity, we conducted the regional functional connectivity analysis by each age group. We calculated the Pearson correlations between the contrast beta values of own-race faces minus that of other-race faces in $\mathrm{Ch} 19 / \mathrm{Ch} 40$ with the contrast beta values between own- and other-race faces in all other channels within each age group. The results were showed on Figure 5. The three age groups showed different pattern of regional functional connectivity: with increased age, the regional functional connectivities between $\mathrm{Ch} 19$ and other channels became more concentrated (see Figure 5a to 5c), showing the brain areas associated with the NORE becoming more specific. Also, with increased age, Channel 40 became more functionally connected with regions in the frontal area (See Figure 5d to 5f).

Correlations between behavioral and NIRS data-To examine the relationship between behavioral and NIRS data, we performed analyses of variance on the NORE (the differences in the [oxy-Hb] activity between own- and other-race faces in Ch19) as the dependent variable, with age (continuous) and the behavioral other-race effect in accuracy (i.e., the difference in accuracy between own- and other-race face recognition; continuous) as independent variables. No significant effects were found.

We performed analyses of variance on the NORE (the differences in the [oxy-Hb] activity between own- and other-race faces in Ch19) as the dependent variable, with age (continuous) and the behavioral ORE in reaction time (i.e., the difference in reaction time between other- and own-race face recognition; continuous) as independent variables. The 
age effects were significant $\left(F(1,71)=4.26, p<.05, \eta^{2}=.06\right)$ : with increased age, the NORE became bigger (part $r=.31, p=.01$ ). The behavioral ORE was also significant ( $F$ $\left.(1,71)=7.59, p<.01, \eta^{2}=.10\right)$ : The greater the children's behavioral ORE in reaction time, the bigger the NORE (part $r=.23, p=.04$ ).

We performed the same ANOVAs with the data in Ch40 and no significant effects were found.

\section{Discussion}

The present study, for the first time, examined the neural correlates of own- and other-race face processing in children between 7 and 13 years of age with the use of fNIRS methodology. We found that (1) behaviorally, children generally did not show significant differences in response accuracy and reaction time when they recognized own- and otherrace faces but their processing performance increased with age; (2) a neural other-race effect (NORE) was found in in Channel 19 (BA9, right MFG/IFG) and Channel 40 (BA18, left cuneus), and this effect changed with age. More specifically, the [oxy-Hb] changes were modestly greater to other-race faces, than own-race faces at younger ages, whereas the [oxy$\mathrm{Hb}$ ] changes were modestly significantly greater to own-race faces than other-race faces in these two cortical areas; (3) regional functional connectivity analysis of the NORE results showed that the Ch19/40 had strong regional functional connectivity with a large swath of the cortical regions. However, with increased age, the regional functional connectivities became more concentrated (Ch19: i.e., reduced number of functionally connected cortical regions) and more connected with the frontal regions (Ch40); (4) We also found significant and positive correlations between the behavioral other-race effect (reaction time) and the neural other-race effect in Ch19. Below we will discuss these major findings.

With regard to the behavioral other-race effect, although the evidence from the existing adult research suggests it to be robust, its effect in middle childhood has not been reliably shown (for a review, see Lee et al., 2011). The present study failed to obtain the behavioral other-race effect with children between 7 and 13 years of age. The null finding might be methodological in nature. Considering the relatively shorter attention span of children and variability of children's ability at face processing in general due to the wide age range used, we had to limit the total amount of time for the experimental procedure. Further, because we used the slow event related design, within the time limit, we had to limit the total number of faces to be remembered and recognized. Consequently, there might be not enough variability in performance for either own- or other-race faces for the other-race effect to emerge.

Nevertheless, despite the fact that children recognized own- and other-race faces equally well, they still showed the neural other-race effect in Channel 19 (BA9, right MFG/IFG) and Channel 40 (BA18, left cuneus). This finding suggests that neural responses might be more sensitive to the processing differences between own- and other-race faces, consistent with the finding by Balas et al. (2011) with infants. The existing adult fMRI studies found that face-race related differential activation in the IFG and MFG is involved in the processing of higher level categorical information associated with the faces as well as familiarity (personal information) (e.g. Feng et al., 2011; Ito \& Bartholow, 2009; Kim et al., 2006). Thus, it is possible that these areas are crucial for children to process faces in general and less familiar face categories in particular. However, no existing adult fMRI studies have found that there was face-race related differential activation in the left cuneus. There was only one study which showed that the right middle fusiform gyrus in a mild cognitively impaired adult group had a higher positive linear correlation with the left cuneus than the healthy control group in own-race face matching tasks (Bokde et al., 2006). The researchers suggested that 
these increases in functional connectivity might be a compensatory process of processing perceptual features of the faces. It is possible that the presenting findings with the left cuneus might also be related to children's reliance on processing face features more generally and the features of other-race faces in particular given the immaturity of their face processing expertise relative to that of adults (Mondloch, Le Grand, \& Maurer, 2010).

Interestingly, the NORE appeared to undergo an age-related shift: at the younger ages, other-race faces elicited modestly greater [oxy-Hb] changes than own-race faces in these two areas, suggesting that young children recruited greater neural resources for processing other-race faces than own-race faces, resulting in a marginally negative neural other-race effect. However, with increased age, own-race faces increasingly elicited modestly greater [oxy-Hb] changes than other-race faces, resulting in a marginally positive neural other race effect. The cognitive mechanisms associated with this age related change are unclear. Given the existing adult fMRI studies regarding the IFG and MFG in terms of face familiarity (e.g. Feng et al., 2011; Ito \& Bartholow, 2009; Kim et al., 2006; Maurer et al., 2007), it is possible that children of different ages had different processing strategies when dealing with faces of familiar and unfamiliar categories. Younger children might have a novelty preference and thus focus on the novel other-race face category more than the more familiar own-race face category. In contrast, older children might have a familiarity preference and thus devote more neural resources to own-race faces, a strategy that is adaptive in children's everyday life as they hardly ever encounter any other-race faces. However, it is worth noting that there was no reliable neural other-race effect in Age Group 2 (from 8.8- to 11.7- yearold). It is likely that with increased age, the familiarity preference for own-race faces increases, whereas the novelty face preference for other-race faces does not decrease. Thus, the two different strategies may co-exist at the same time and together contributed to the lack of a NORE in this age group.

Furthermore, we found significant and positive correlations between the behavioral ORE (reaction time) and the NORE in Channel 19. That is, the greater children's NORE (neural activity between own minus other-race face) was, the bigger the behavioral ORE in reaction time (the difference in reaction time between other- and own-race face recognition). Thus, that the involvement of these frontal areas (IFG/MFG) increased with age may support the suggestion that older children devoted greater neural resources to processing own-race faces than other-race ones.

The GLM results can only describe the activation between the two conditions but not the functional interactions between different areas. Some studies have shown that functional interaction between regions of a cognitive network is also a possible marker for detection of the neural correlates of cognitive processing (Bokde et al., 2006). We found that Channels $19 / 40$ had high linear correlations with a large swath of the cortical regions in terms of the neural other-race effect. The functional interaction between Ch19/40 and other cortical areas was robust even after the effect of age was partialled out. Further, the correlations were all positive. Thus, the greater the neural other-race effects in Channels 19/40, the greater the same effect in the related cortical regions, suggesting that the differences in the amount of neural resources devoted to processing own- and other-race faces were similarly across a large swath of cortical regions. These results are largely in line with the idea that the processing differences between own- and other-race faces involve both bottom-up and topdown processing (Ito \& Bartholow, 2009). Also, it is worth noting that there were agerelated changes in regional functional connectivities between Channels 19/40 and other cortical areas. Specifically, the connectivities of Channel 19 to other cortical regions became more concentrated and limited to fewer channels as age increased. This reduced regional functional connectivity may be due to the fact that as children's face processing expertise increases with age, they became more efficient and domain-specific in using the frontal 
neural resources in processing the own- and other-race faces. This finding is in line with a general finding in the developmental fMRI literature that with increased age, the activations of children's face processing develops from more diffused to more focal (Aylward et al., 2005; Passarotti et al., 2003; Passarotti et al., 2007; for a review, Lee et al., 2011).

Interestingly, Ch40's connectivity to the frontal regions increased with increased age. Thus, with increased age, the integration of own- and other-race face processing appeared to improve along the anterior-posterior axis, which may reflect the age-related enhancement in coupling between the core face processing network and the extended face processing network (Haxby et al., 2000).

\section{Limitations}

Due to the limitation of the cortical depth that the NIRS signal can be detected by the optical detectors on the scalp, we could only assess the cortical regions involved in face-race processing that are close to the cortical surface. For this reason, we were unable to examine the roles of some important brain areas such as the ACC, amygdala, and the FFA. In addition, due to the limited number of optodes (46 channels) that we could use, neural activities in some cortical regions not covered by these channels were unfortunately missed. Thus, future fMRI studies are needed to replicate and extend the current findings and to uncover other important cortical and subcortical regions involved in own- and other-race face processing in childhood. In addition, there is also a limitation to our paradigm. Due to the limited number of faces to be remembered and recognized, there might be not enough variability in behavioral performance. As a result, our task failed to reveal a behavioral other-race effect, a highly robust phenomenon found mostly with adults. Thus, more sensitive behavioral paradigms are needed so as to obtain a behavioral other-race effect, which in turn might have better sensitivities to reveal the neural other-race effect than the present study. Furthermore, in the present study, we only recruited children 7-13 years of age as participants, whereas the effect has been reported in preschoolers and even infants. Future fNIRS studies need to extend to these younger age groups so as to obtain a comprehensive picture of the development of the other-race effect in behavior and in neural responsitivity as the fNIRS methodology has been shown to be well suited for studying face processing in even very young infants (Kobayashi et al., 2012; Nakato et al., 2009; Otsuka et al., 2007). Last but not least, future studies need to assess the accuracy and validity of using the probabilistic registration method (Singh et al., 2005) to estimate child MNI coordinates and thus the exact MNI locations of the optical channels reported here need to be interpreted with caution.

\section{Summary}

The present study for the first time used the functional Near-infrared Spectroscopy (fNIRS) methodology to investigate the neural correlates of children's own- and other-race processing. An old-new paradigm was used to assess children's recognition ability of ownand other-race faces. The results showed that children generally did not show significant differences in response accuracy and reaction time when they recognized own- and otherfaces but their processing performance increased with age. However, in terms of neural responses, other-race faces elicited significantly greater [oxy-Hb] changes than own-race faces in Ch19 (BA9, right MFG/IFG) and Channel 40 (BA18, left cuneus). Further, with increased age, the [oxy-Hb] activity differences between own- and other-race faces, or the NORE, underwent significant changes in these two cortical areas: at younger ages, the neural response to the other-race faces was greater than that to the own-race faces, but with increased age, the neural response to the own-race faces became increasing greater than that to the other-race faces. Moreover, Channels 19/40 showed strong regional functional connectivity with a large swath of the cortical regions in terms of the neural other-race effect and these regional functional connectivities also went significant changes with increased 
age. We also found significant and positive correlations between the behavioral ORE (reaction time) and the NORE in Channel 19. Our findings taken together suggest that children, like adults, devote different amounts of neural resources to processing own- and other-race faces but the size and direction of the neural other-race effect change with increased age.

\section{Supplementary Material}

Refer to Web version on PubMed Central for supplementary material.

\section{References}

Anzures G, Quinn PC, Pascalis O, Slater AM, Lee K. Categorization, categorical perception, and asymmetry in infants' representation of face race. Developmental Science. 2010; 13:553-564. [PubMed: 20590720]

Anzures G, Quinn PC, Pascalis O, Slater AM, Tanaka JW, Lee K. The developmental origins of the other-race effect. Current Directions in Psychological Science. (in press).

Anzures G, Kelly DJ, Pascalis O, Quinn PC, Slater AM, de Viviés Xavier, Lee K. Own- and other-race face identity recognition in children: The effects of pose and feature composition. Developmental Psychology. (in press).

Aylward EH, Park JE, Field KM, Parsons AC, Richards TL, Cramer SC, Meltzoff AN. Brain activation during face perception: evidence of a developmental change. Journal of Cognitive Neuroscience. 2005; 17:308-319. [PubMed: 15811242]

Bahrick HP, Bahrick PO, Wittlinger RP. Fifty years of memory for names and faces: A cross-sectional approach. Journal of Experimental Psychology: General. 1975; 104:54-75.

Balas B, Westerlund A, Hung K, Nelson CA. Shape, color, and the other-race effect in the infant brain. Developmental Science. 2011; 14:892-900. [PubMed: 21676108]

Bar-Haim Y, Ziv T, Lamy D, Hodes RM. Nature and nurture in own-race face processing. Psychological Science. 2006; 17:159-163. [PubMed: 16466424]

Bokde ALW, Meindl T, Pechler S, Born C, Faltraco F, Teipel SJ, Mo H. Functional connectivity of the fusiform gyrus during a face-matching task in subjects with mild cognitive impairment. Brain. 2006; 129:1113-1124. [PubMed: 16520329]

Caharel S, Montalan B, Fromager E, Bernard C, Lalonde R, Mohamed R. Other-race and inversion effects during the structural encoding stage of face processing in a race categorization task: An event-related brain potential study. International Journal of Psychophysiology. 2011; 79:266-271. [PubMed: 21055428]

Caldara R, Rossion B, Bovet P, Hauert C. Event-related potentials and time course of the 'other-race' face classification advantage. Cognitive Neuroscience and Neuropsychology. 2004; 15:905-910.

Chance JE, Turner AL, Goldstein AG. Development of differential recognition for own- and other-race faces. The Journal of Psychology. 1982; 112:29-37. [PubMed: 7143272]

Cope M, Delpy DT. System for long-term measurement of cerebral blood and tissue oxygenation on newborn infants by near infra-red transillumination. Medical \& Biological Engineering \& Computing. 1988; 26:289-294. [PubMed: 2855531]

Cunningham WA, Johnson MK, Raye CL, Gatenby JC, Gore JC, Banaji MR. Separable neural components in the processing of black and white faces. Psychological Science. 2004; 15:806-813. [PubMed: 15563325]

Esterman M, Yantis S. Perceptual expectation evokes category-selective cortical activity. Cerebral Cortex. 2010; 20:1245-1253. [PubMed: 19759124]

Feinman S, Entwisle DR. Children's ability to recognize other children's faces. Child Development. 1976; 47:506-510. [PubMed: 1269316]

Feng L, Liu J, Wang Z, Li J, Li L, Ge L, Lee K. The other face of the other-race effect: an fMRI investigation of the other-race face categorization advantage. Neuropsychologia. 2011; 49:37393749. [PubMed: 21971308] 
Ferguson KT, Kulkofsky S, Casasola M, Cashon C. The development of specialized processing of own-race faces in infancy. Infancy. 2009; 14:263-284.

Ge L, Zhang H, Wang Z, Quinn PC, Pascalis O, Kelly D, Lee K. Two faces of the other-race effect: Recognition and categorisation of Caucasian and Chinese faces. Perception. 2009; 38:1199-1210. [PubMed: 19817152]

Golby AJ, Gabrieli JDE, Chiao JY, Eberhardt JL. Differential responses in the fusiform region to same-race and other-race faces. Nature Neuroscience. 2001; 4:845-850.

Goodman GS, Sayfan L, Lee JS, Sandhei M, Walle-Olsen A, Magnussen S, Arredondo P. The development of memory for own- and other-race faces. Journal of Experimental Child Psychology. 2007; 98:233-242. [PubMed: 17928001]

Hancock KJ, Rhodes G. Contact, configural coding and the other-race effect in face recognition. British Journal of Psychology. 2008; 99:45-56. [PubMed: 17535471]

Haxby JV, Hoffman EA, Gobbini MI. The distributed human neural system for face perception. Trends in Cognitive Sciences. 2000; 4:223-233. [PubMed: 10827445]

Hayden A, Bhatt RS, Joseph JE, Tanaka JW. The other-race effect in infancy: Evidence using a morphing technique. Infancy. 2007; 12:95-104.

Hayden A, Bhatt RS, Zieber N, Kangas A. Race-based perceptual asymmetries underlying face processing in infancy. Psychonomic Bulletin \& Review. 2009; 16:270-275. [PubMed: 19293093]

Hayward WG, Rhodes G, Schwaninger A. An own-race advantage for components as well as configurations in face recognition. Cognition. 2008; 106:1017-1027. [PubMed: 17524388]

Homae F, Watanabe H, Nakano T, Taga G. Prosodic processing in the developing brain. Neuroscience Research. 2007; 59:29-39. [PubMed: 17590470]

Hugenberg K, Young SG, Bernstein MJ, Sacco DF. The categorization-individuation model: An integrative account of the other-race recognition deficit. Psychological Review. 2010; 117:11681187. [PubMed: 20822290]

Ito TA, Bartholow BD. The neural correlates of race. Trends in Cognitive Science. 2009; 13:524-531.

Ito TA, Urland GR. The influence of proessing objectives on the perception of faces: An ERP study of race and gender perception. Cognitive, Affective, \& Behavioral Neuroscience. 2005; 5:21-36.

Jang KE, Tak S, Jung J, Jang J, Jeong Y, Ye JC. Wavelet minimum description length detrending for near-infrared spectroscopy. Journal of biomedical optics. 2009; 14:034004. [PubMed: 19566297]

Kanwisher N, Yovel G. The fusiform face area: A cortical region specialized for the perception of faces. Philosophical Transactions of the Royal Society B. 2006; 361:2109-2128.

Kelly DJ, Liu S, Lee K, Quinn PC, Pascalis O, Slater AM, Ge L. Development of the other-race effect in infancy: Evidence towards universality? Journal of Experimental Child Psychology. 2009; 104:105-114. [PubMed: 19269649]

Kelly DJ, Quinn PC, Slater AM, Lee K, Ge L, Pascalis O. The other-race effect develops during infancy: Evidence of perceptual narrowing. Psychological Science. 2007; 18:1084-1089. [PubMed: 18031416]

Kelly DJ, Quinn PC, Slater AM, Lee K, Gibson A, Smith M, Pascalis O. Three-month-olds, but not newborns, prefer own-race faces. Developmental Science. 2005; 8:31-36.

Kim J, Yoon H, Kim B, Jeun S, Jung S, Choe B. Racial distinction of the unknown facial identity recognition mechanism by event-related fMRI. Neuroscience Letters. 2006; 397:279-284. [PubMed: 16446032]

Kobayashi M, Otsuka Y, Nakato E, Kanazawa S, Yamaguchi MK, Kakigi R. Do infants recognize the Arcimboldo images as faces? Behavioral and near-infrared spectroscopic study. Journal of Experimental Child Psychology. 2012; 111:22-36. [PubMed: 21875715]

Lee, K.; Anzures, G.; Quinn, PC.; Pascalis, O.; Slater, A. Development of face processing expertise. In: Calder, A.; Rhodes, G.; Haxby, JV.; Johnson, M., editors. Handbook of Face Perception. Oxford, UK: Blackwell Publishing; 2011. p. 753-778.

Li J, Liu J, Liang J, Zhang H, Zhao J, Rieth CA, Lee K. Effective connectivities of cortical regions for top-down face processing: A dynamic causal modeling study. Brain Research. 2010; 1340:40-51. [PubMed: 20423709] 
Lieberman MD, Hariri A, Jarcho JM, Eisenberger NI, Bookheimer SY. An fMRI investigation of racerelated amygdala activity in African-American and Caucasian-American individuals. Nature Neuroscience. 2005; 8:720-722.

Maki A, Yamashita Y, Ito Y, Watanabe E, Mayanagi Y, Koizumi H. Spatial and temporal analysis of human motor activity using noninvasive NIR topography. Medical physics. 1995; 22:1997-2005. [PubMed: 8746704]

Maurer D, O'Craven KM, Grand RL, Mondloch CJ, Springer MV, Lewis TL, Grady CL. Neural correlates of processing facial identity based on features versus their spacing. Neuropsychologia. 2007; 45:1438-1451. [PubMed: 17204295]

Meissner CA, Brigham JC. Thirty years of investigating the own-race bias in memory for faces: A meta-analytic review. Psychology, Public Policy, and Law. 2001; 7:3-35.

Mondloch CJ, Elms N, Maurer D, Rhodes G, Hayward WG, Tanaka JW, Zhou G. Processes underlying the cross-race effect: An investigation of holistic, featural, and relational processing of own-race versus other-race faces. Perception. 2010; 39:1065-1085. [PubMed: 20942358]

Mondloch, CJ.; Le Grand, R.; Maurer, D. Development of expertise in face recognition. In: Gauthier, I.; Tarr, MJ.; Bub, D., editors. Perceptual expertise; Bridging brain and behavior. Oxford University Press; 2010. p. 67-106.

Morita T, Itakura S, Saito D, Nakashita S, Harada T, Kochiyama T, Sadato N. The role of the right prefrontal cortex in self-evaluation of the face: A functional magnetic resonance imaging study. Journal of Cognitive Neuroscience. 2008; 20:342-355. [PubMed: 18275339]

Nakato E, Otsuka Y, Kanazawa S, Yamaguchi MK, Watanabe S, Kakigi R. When do infants differentiate profile face from frontal face? A near-infrared spectroscopic study. Human Brain Mapping. 2009; 30:462-472. [PubMed: 18095284]

Natu V, Raboy D, O'Toole AJ. Neural correlates of own- and other-race face perception: Spatial and temporal response differences. NeuroImage. 2010; 54:2547-2555. [PubMed: 20937393]

Otsuka Y, Nakato E, Kanazawa S, Yamaguchi MK, Watanabe S, Kakigi R. Neural activation to upright and inverted faces in infants measured by near infrared spectroscopy. NeuroImage. 2007; 34:399-406. [PubMed: 16997577]

Passarotti AM, Paul BM, Bussiere JR, Buxton RB, Wong E, Stiles J. The development of face and location processing: an fMRI study. Developmental Science. 2003; 6:100-117.

Passarotti AM, Smith J, DeLano M, Huang J. Developmental differences in the neural bases of the face inversion effect show progressive tuning of face-selective regions to the upright orientation. NeuroImage. 2007; 34:1708-1722. [PubMed: 17188904]

Pezdek K, Blandon-Gitlin I, Moore C. Children's face recognition memory: More evidence for the cross-race effect. Journal of Applied Psychology. 2003; 88:760-763. [PubMed: 12940414]

Rhodes G, Hayward WG, Winkler C. Expert face coding: Configural and component coding of ownrace and other-race faces. Psychonomic Bulletin \& Review. 2006; 13:499-505. [PubMed: 17048737]

Sangrigoli S, de Schonen S. Recognition of own-race and other-race faces by three-month-old infants. Journal of Child Psychology and Psychiatry. 2004a; 45:1219-1227. [PubMed: 15335342]

Sangrigoli S, de Schonen S. Effect of visual experience on face processing: A developmental study of inversion and non-native effects. Developmental Science. 2004b; 7:74-87. [PubMed: 15323120]

Sangrigoli S, Pallier C, Argenti AM, Ventureyra VAG, de Schonen S. Reversibility of the other-race effect in face recognition during childhood. Psychological Science. 2005; 16:440-444. [PubMed: 15943669]

Scott LS, Pascalis O, Nelson CA. A domain-general theory of the development of perceptual discrimination. Current Directions in Psychology Science. 2007; 16:197-201.

Singh AK, Okamoto M, Dan H, Jurcak V, Dan I. Spatial registration of multichannel multi-subject fNIRS data to MNI space without MRI. NeuroImage. 2005; 27:842-851. [PubMed: 15979346]

Suda M, Takei Y, Aoyama Y, Narita K, Sato T, Fukuda M, Mikuni M. Frontopolar activation during face-to-face conversation: An in situ study using near-infrared spectroscopy. Neuropsychologia. 2010; 48:441-447. [PubMed: 19819248] 
Tak S, Yoon SJ, Jang J, Yoo K, Jeong Y, Ye JC. Quantitative analysis of hemodynamic and metabolic changes in subcortical vascular dementia using simultaneous near-infrared spectroscopy and fMRI measurements. NeuroImage. 2011; 55:176-84. [PubMed: 21094685]

Van Bavel JJ, Packer DJ, Cunningham WA. The neural substrates of in-group bias: a functional magnetic resonance imaging investigation. Psychological Science. 2008; 19:1131-1139. [PubMed: 19076485]

Walker PM, Hewstone M. A perceptual discrimination investigation of the own-race effect and intergroup experience. Applied Cognitive Psychology. 2006; 20:461-475.

Walker PM, Tanaka JW. An encoding advantage for own-race versus other-race faces. Perception. 2003; 32:1117-1125. [PubMed: 14651324] 


\section{Highlights}

- We examined neural correlates of children's other-race face effect using fNIRS.

- $\quad[O x y-H b]$ activities showed a neural other-race effect.

- With increased age, the neural other-race effect increased significantly.

- The correlations between behavioral and neural other-race effect were significant.

- Children used neural resources differently to process own- and other-race faces. 


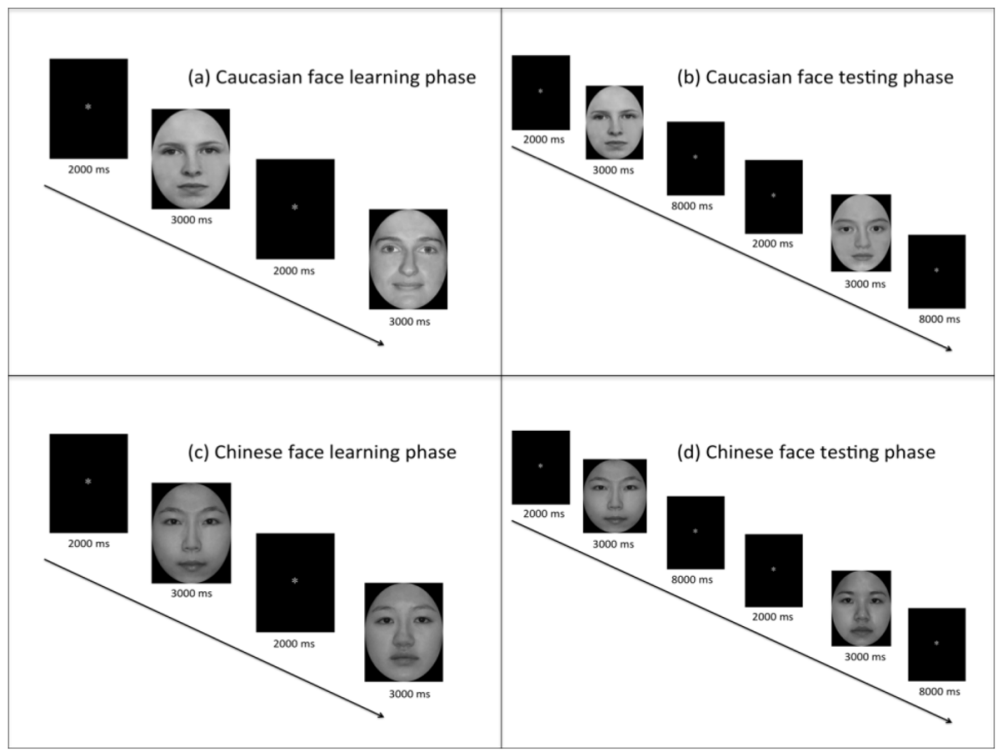

Figure 1.

The experimental design: (a) Caucasian face learning phase; (b) Caucasian face testing phase; (c) Chinese face learning phase; (d) Chinese face testing phase. 


\section{a}

left frontal

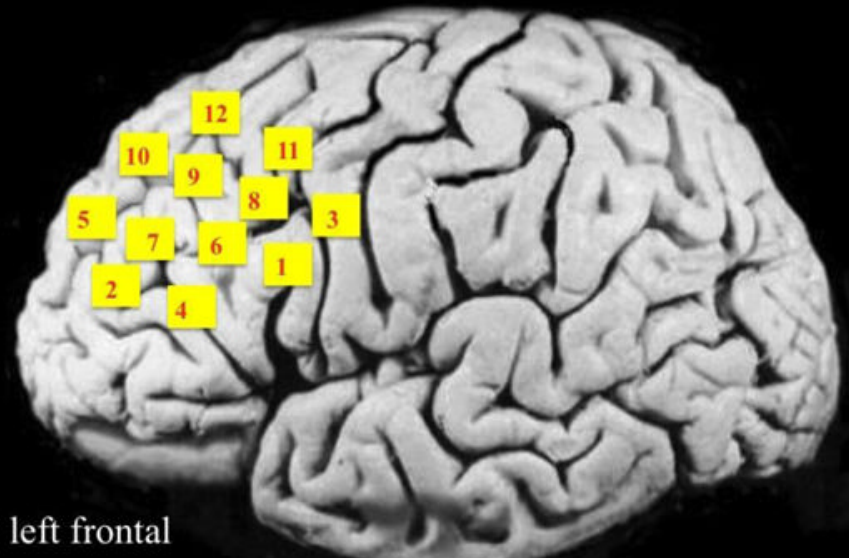

Fig. 2a

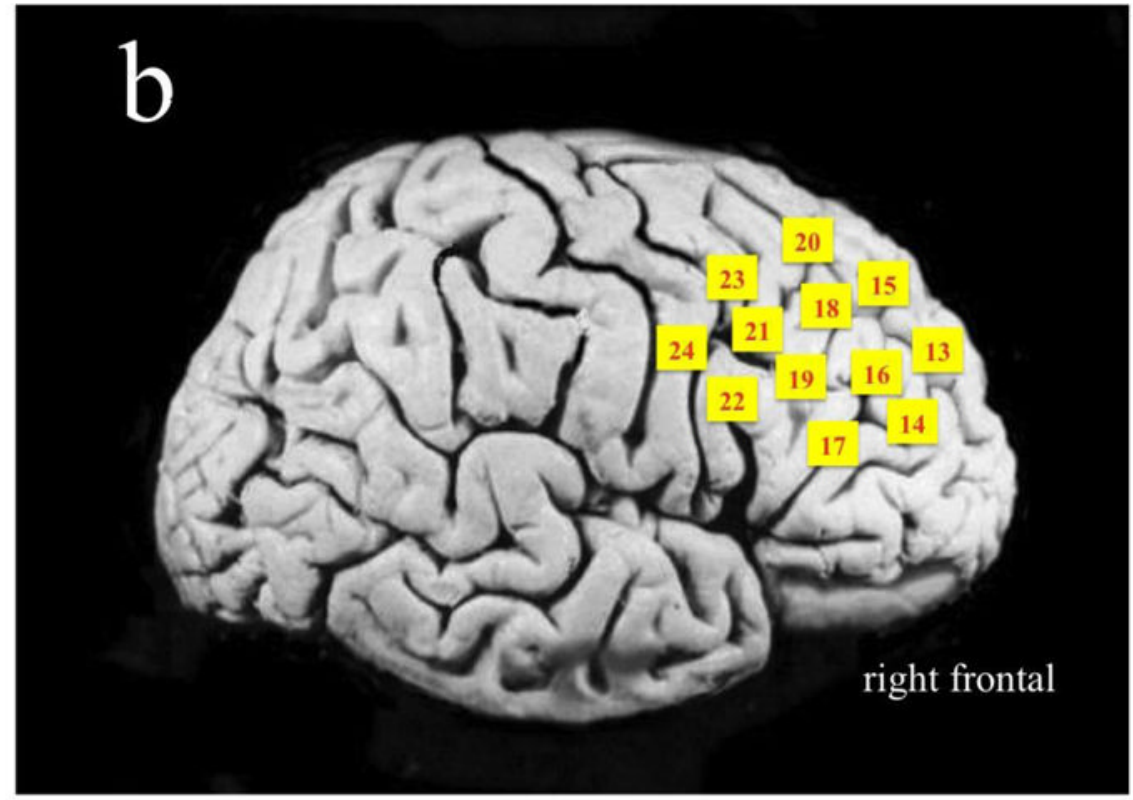

Fig. $2 b$ 


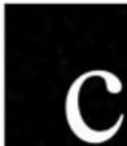

right

Fig. 2c

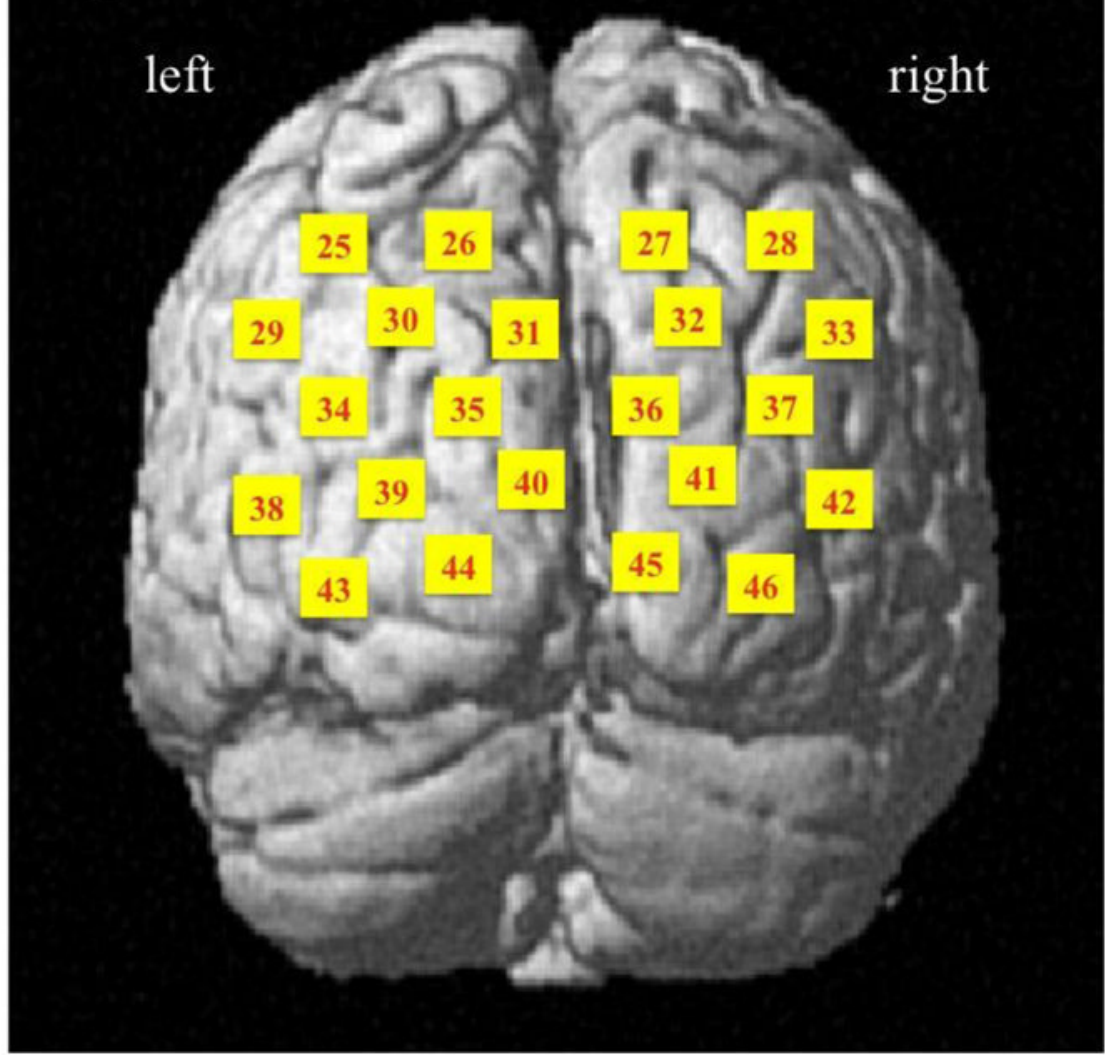

\section{Figure 2.}

Estimated cortical locations of the 46 NIRS channels. 


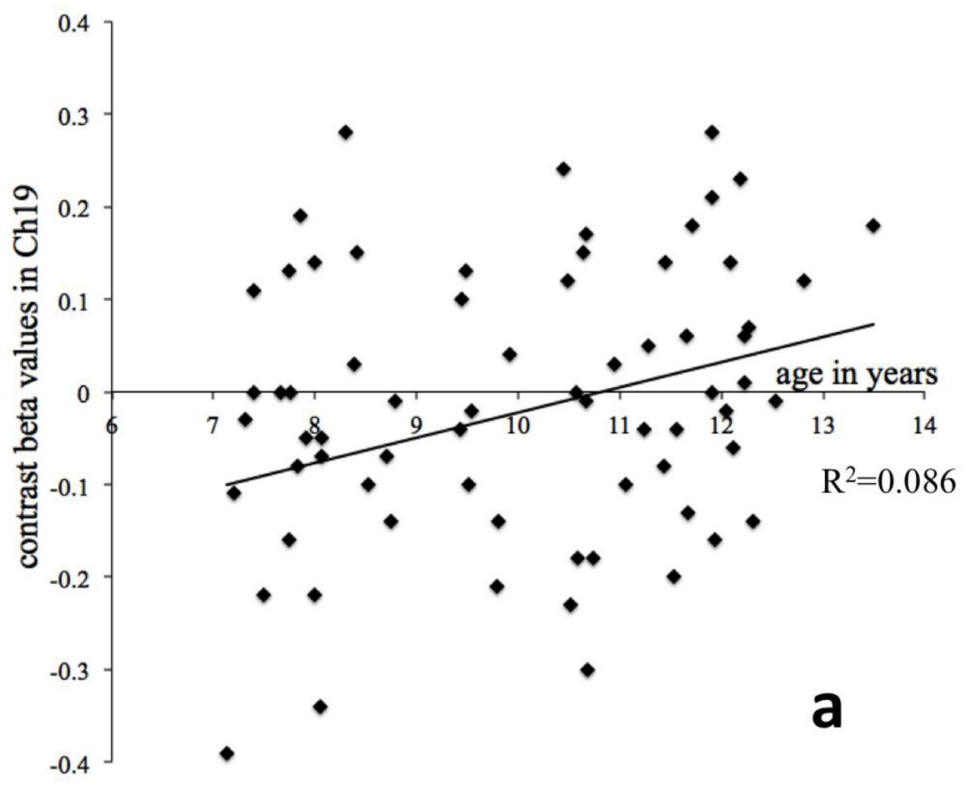

Fig. 3a

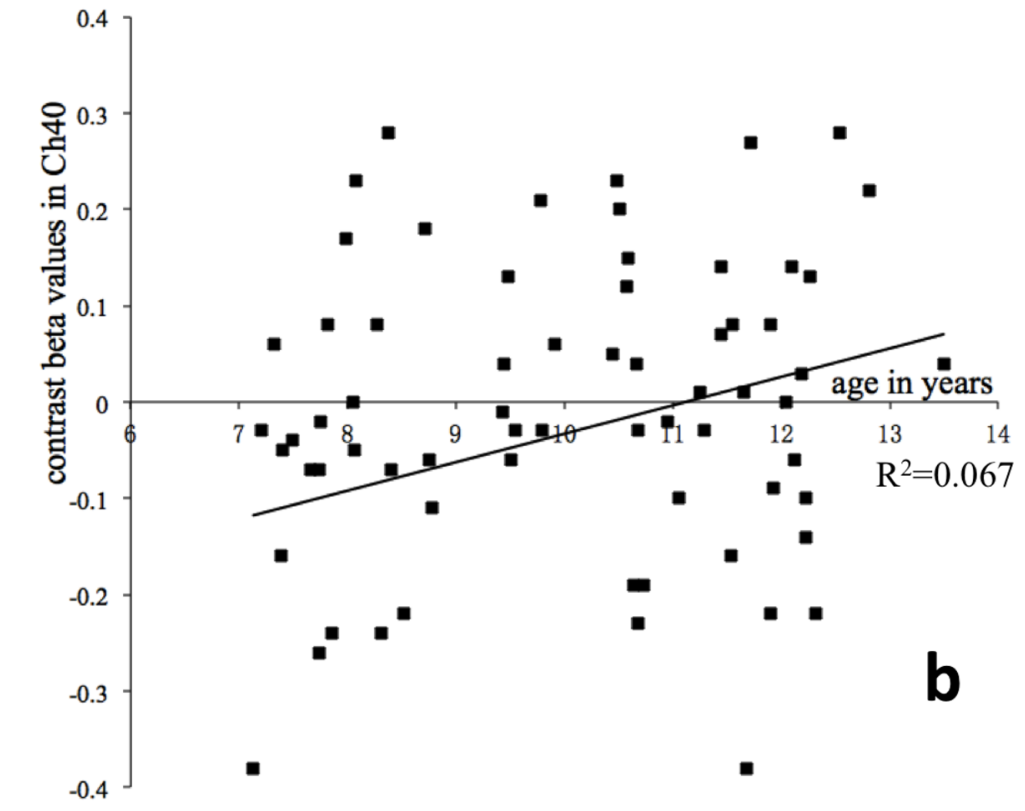

Fig. $3 b$

Figure 3.

Correlations of the beta values of the significant channels and age in years 


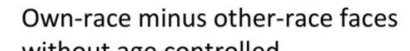
without age controlled

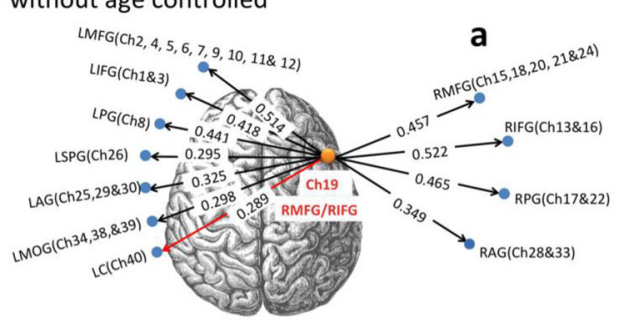

Own-race minus other-race faces with age controlled

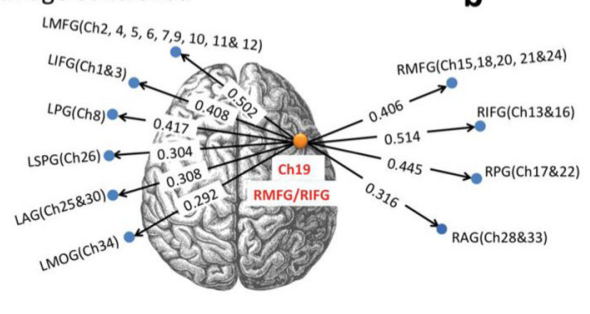

Own-race minus other-race faces without age controlled

C

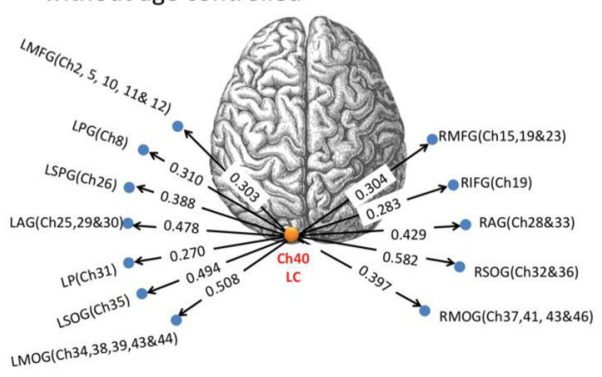

Own-race minus other-race faces with age controlled

d

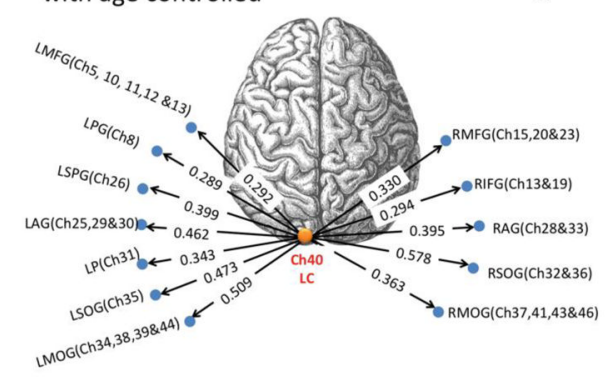

Figure 4.

Correlations of beta values in Channels 19/40 with other brain areas with all children's data combined. 

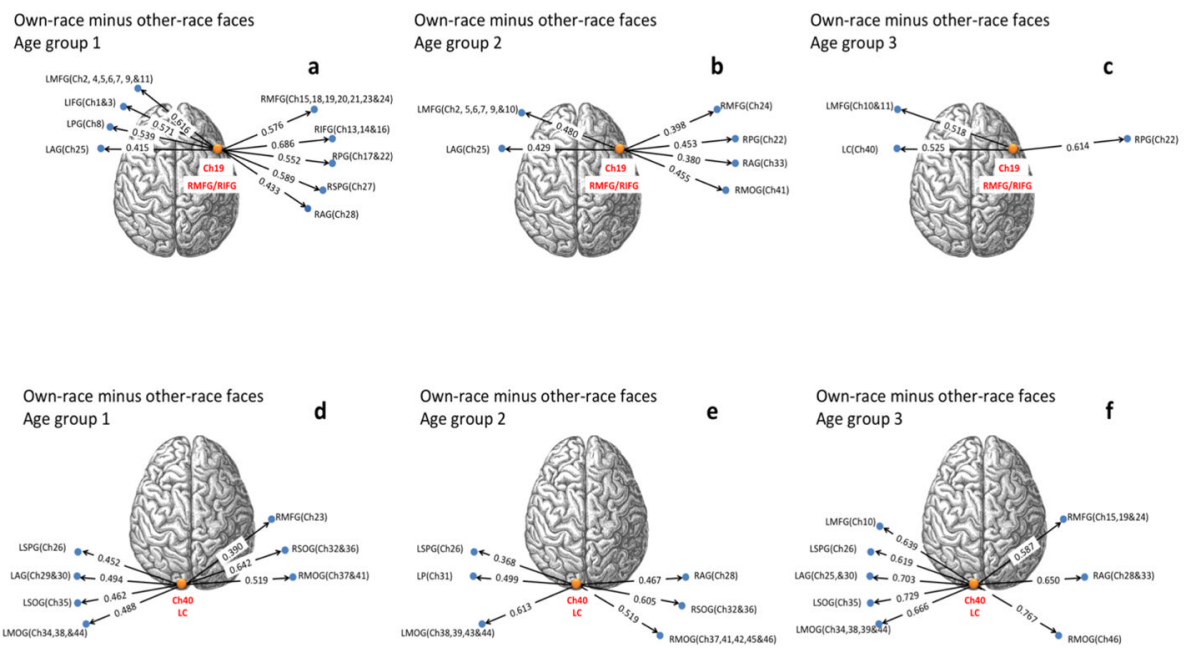

Figure 5.

Correlations of beta values in Channels 19/40 with other brain areas in different age groups. 


\section{Table 1}

Means (SD) of children's recognition accuracy and correct reaction time of own- and other-race faces

\begin{tabular}{ccc}
\hline & Other-race faces & Own-race faces \\
\hline Accuracy & $.72(.12)$ & $.74(.12)$ \\
Reaction time $(\mathrm{ms})$ & $1790(400)$ & $1755(348)$ \\
\hline
\end{tabular}



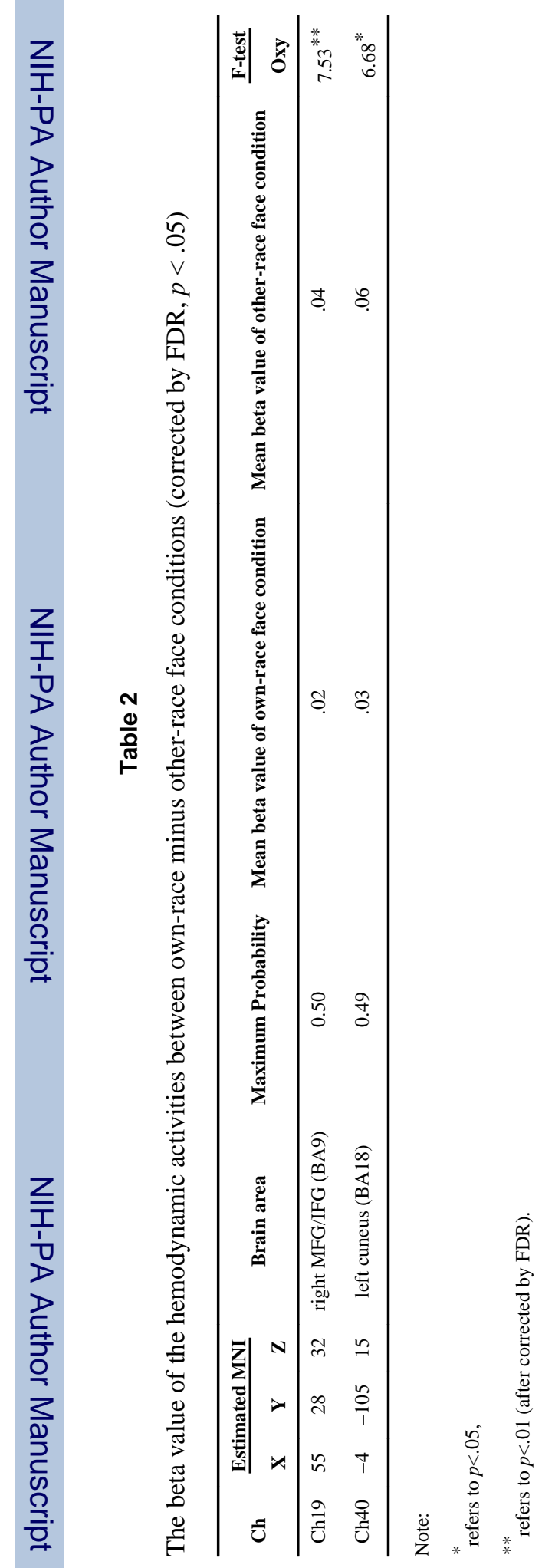

Neuroimage. Author manuscript; available in PMC 2015 January 15. 